\title{
Chili und Ribiselkuchen: Gemeinschaftsgärten im Wiener Gemeindebau als gemeinschaftliches Außenhaus
}

\author{
Rita Mayrhofer*
}

\section{Zusammenfassung}

In Wien sind in den letzten zehn Jahren über 70 Gemeinschaftsgärten entstanden. Bislang waren es vor allem Menschen mit überdurchschnittlicher Bildung und kreativen Berufen (creative class), die sich ihren Traum vom gemeinschaftlichen Gärtnern erfüllen konnten. Menschen mit geringem Bildungsgrad und sozial benachteiligte Gruppen sind in den Wiener Gemeinschaftsgärten hingegen bislang kaum vertreten. Diesem class bias entgegenzuwirken und gleichzeitig ungenutzte Flächenpotenziale zu aktivieren, waren die Ziele eines Pilotprojekts, das in den Jahren 2009 bis 2011 im Wiener Gemeindebau umgesetzt wurde. Basierend auf den Daten einer Aktionsforschung sowie aktueller empirischer Erhebungen geht der Text auf die Rahmenbedingungen, den partizipativen Planungsprozess und die Wirkungen eines selbstbestimmten Gemeinschaftsgartens im sozialen Wohnbau ein. Mit der Transformation des funktionellen Abstandsgrüns in ein gemeinschaftliches Außenhaus (Hülbusch 1978) entstanden neue „Handlungs-Freiräume“ im Sinne einer vita activa (Arendt 1969). Das Beispiel zeigt, dass soziale Inklusion durch Gemeinschaftsgärten im sozialen Wohnbau möglich ist. Besonders wichtig sind dabei freiraumplanerische Organisationsprinzipen und ein auf Selbstbestimmung abzielender Beteiligungsprozess.

Schlagwörter: Community Gardening, Sozialer Wohnbau, Wien, Außenhaus, Vita activa

\section{Chili and Ribiselkuchen: Thinking Community Gardening in Viennese municipal housing as shared Außenhaus}

\section{Abstract}

Over the past ten years more than 70 community gardens have been established in the city of Vienna. So far, mainly people with above-average education and creative professions (creative class) have been able to fulfil their dream of community gardening. In contrast, people with a low level of education and socially disadvantaged groups are hardly represented in Viennese Community Gardens so far. To counteract this class bias and to activate unused spatial potentials at the same time were the aims of a pilot project that took place in the years 2009 to 2011 in a social housing area in Vienna. Based on data from action research and an empirical survey of the current situation, the text deals with the framework conditions, the participatory process and the effects of an autonomous community garden in the context of social housing. With the transformation of the functional distance green into a communal Außenhaus (Hülbusch 1978), new "spaces for action" in the sense of a vita activa were created (Arendt 1969). This case shows that community gardens can foster social inclusion in social housing areas. Particularly important are the principles of open space planning and a participation process aiming at self-determination.

Keywords: Community gardening, social housing, pilot project, vita activa

Rita Mayrhofer, Institut für Landschaftsplanung, Universität für Bodenkultur Wien, E-Mail: rita.mayrhofer@boku.ac.at Dieser Artikel beruht auf theoretischen Impulsen und anregenden Diskussionen mit Andrea Kölzer und Matthias Kurowski. Ich danke Michael Ornetzeder für das kritische Hinterfragen meiner Erkenntnisse, meinen Institutskolleg*innen fürs Kommentieren und Korrigieren, den beiden anonymen Gutachter*innen für die wertvollen Hinweise zur Verbesserung des Textes, sowie den Frauen vom Verein Wirbel und den Gärtner*innen für die jahrelange Zusammenarbeit. 


\section{Einleitung}

Drei Frauen sitzen am Tisch in der Vormittagssonne und essen gemeinsam Schafkäse, Dolma und Pide. Dazu gibt es Salatblätter und Kräuter aus den Beeten hinter ihnen. In einem anderen Teil des Gartens erntet eine Frau gerade Chilischoten. Beim Kompost neben der Hütte zerkleinert ein Mann einige Äste. Eine weitere Frau kommt durchs Gartentor. Sie hat selbst gemachten Ribiselkuchen und Kaffee mitgebracht und setzt sich zur Gruppe unter dem Apfelbaum. Das ist eine durchaus typische Situation im Gemeinschaftsgarten eines Wiener Gemeindebaus.

In Wien sind in den letzten zehn Jahren über 70 gemeinschaftliche Gartenprojekte entstanden. Lange Wartelisten für zukünftige Projekte deuten darauf hin, dass die Nachfrage ungebrochen ist. ${ }^{1}$ In wachsenden Städten wie Wien stehen dem Wunsch nach neuen Gemeinschaftsgärten jedoch eine Reihe von Hindernissen gegenüber. Geeignete Flächen sind rar, im öffentlichen Raum befinden sie sich meist in Konkurrenz zu anderen Nutzungen, längerfristige Nutzungsverträge werden kaum vergeben und es braucht auch einiges an Know-how und Zeit, um eine Garteninitiative $\mathrm{zu}$ starten (Müller 2011). Seit dem Jahr 2010 fördert die Gemeinde Wien die Errichtung von Gemeinschaftsgärten, sowohl finanziell als auch mit direkten Beratungsund Unterstützungsleistungen durch stadtteilbezogene Organisationen $^{2}$ (Exner et al. 2016). Diese kommunal initiierten und verwalteten Gartenprojekte machen inzwischen etwa zwei Drittel aller Gemeinschaftsgärten in Wien aus.

Jedoch haben nicht alle Menschen die gleiche Chance der Teilhabe und Mitgestaltung an dieser neuen, sehr beliebten Form des Gärtnerns. Eine aktuelle Untersuchung zeigt, dass Gemeinschaftsgärten in Wien einen class character haben, der ihre Erscheinungsform bestimmt. Unter den Gärtnerinnen dominiert eine kulturelle Elite, eine creative class aus Personen mit hohem Bildungsgrad und überdurchschnittlich großem sozialem und z.T. auch wirtschaftlichem Kapital. Angehörige von Minderheiten oder sozial benachteiligten Gruppen sind in den Wiener

1 https://gartenpolylog.org/gartenkarte [29.11.2019]

2 Wiener Gebietsbetreuung (seit 1974 ein Element einer partizipativen Politik in Wien, organisiert als dezentrale Körperschaft der Gemeinde Wien) und die Lokale Agenda 21 (seit 2001 eine weitere dezentrale Körperschaft in Wien für eine nachhaltige Entwicklung durch Partizipation) siehe auch Exner et al. (2016).
Gemeinschaftsgärten deutlich unterrepräsentiert (Exner/Schützenberger 2018).

Gemeinschaftsgärten in den sozialen Wohnbau zu integrieren kann dieser sozialen Exklusivität entgegenwirken und gleichzeitig dazu beitragen, ein neues Potenzial an Flächen $\mathrm{zu}$ erschließen. In der wissenschaftlichen Literatur wurde das Thema Gemeinschaftsgärten im sozialen Wohnbau bisher kaum berücksichtigt (Lewis 1972; Kaplan/Kaplan 1989; Wakefield et al. 2007; Cumbers et al. 2018). Doch gerade in diesem Kontext erscheint das gemeinschaftliche Gärtnern aus mehreren Gründen sinnvoll. Die Nähe zwischen Wohnung und Garten unterstützt die Praxis des Gärtnerns, aufgrund der sozialräumlichen Veränderungsprozesse im sozialen Wohnbau von der Arbeiter*innenwohnsiedlung zum Wohnort für benachteiligte Gruppen (Reinprecht 2007) kommen andere soziale Milieus in den Genuss der Vorteile und die weitgehend ungenutzten parkartigen Freiräume der Gemeindebauten stehen im Vergleich zum öffentlichen Raum weniger in Konkurrenz mit anderen Nutzungen.

Um Gemeinschaftsgärten auch im Wiener Gemeindebau ${ }^{3} \mathrm{zu}$ etablieren und dieses Potenzial zu erschließen, initiierte ein interdisziplinäres Team 2009 das Pilotprojekt Nachbarschaftsgarten Roda-Roda ${ }^{4}$. Es war einer der ersten aus kommunalen Mitteln geförderten Gemeinschaftsgärten in Wien (Emprechtinger et al. 2010).

Dieser Artikel setzt sich anhand einer Fallstudie mit folgender Frage auseinander: Welche Prinzipien und Prozesse ermöglichen eine Transformation des Abstandsgrüns im sozialen Wohnbau in einen Raum zum Tätigsein? Drei Aspekte sind bei der Beantwortung dieser Frage von besonderer Bedeutung. Zunächst erfordert ein gutes oder tätiges Leben im Sinne einer vita activa nach Hannah Arendt (1969) eine geeignete räumliche Rahmenstruktur. Von dieser im Zusammen-

3 Wiener Gemeindebau: aktuell 2.30o kommunale Wohngebäude, rund 500.000 Bewohner*innen (ca. ein Viertel der Gesamtbevölkerung Wiens), 610 Hektar Grünflächen (Der Wiener Gemeindebau. Geschichte, Daten, Fakten https://www.wienerwohnen.at/dokumente-downloads.html [29.11. 2019].

4 Das Pilotprojekt (2009 bis 2011): durchgeführt vom Verein WIRBEL (Heide Studer, Susanne Staller, Sonja Gruber, Kirsten Förster, Rita Mayrhofer), gefördert von Wiener Wohnen und der Magistratsabteilung 57. Begleitforschung und Evaluierung: durchgeführt vom Verein WIRBEL in Zusammenarbeit mit dem wissenschaftlichen Forschungszentrum KOSAR, gefördert von der Wiener Wohnbauforschung (MA 50). 
hang mit dem Phänomen Gemeinschaftsgärten neuen Perspektive gehen wichtige Impulse für die Planung aus. In weiterer Folge geht es darum, die Räume zu benennen, die dabei entstehen. Das Planungsprinzip „Innenhaus und Außenhaus“ (Hülbusch 1978) eröffnet dazu eine Perspektive, weil es gezielt baulich-räumliche, soziale und ökonomische Rahmenstrukturen für ein gutes Leben im Sinne einer Subsistenzperspektive (Bennholdt-Thomsen 2014) und des postpatriarchalen Denkens (Knecht et al. 2015; Hirsch 1985) thematisiert. Schließlich geht es um die Frage, wie ein von außen initiierter, auf die Umverteilung von Macht und Verantwortung ausgerichteter Beteiligungsprozess gestaltet sein muss, um soziale Inklusivität in Gemeinschaftsgärten zu fördern. Darüber hinaus trägt die Betrachtung des neuen Freiraumtyps Gemeinschaftsgarten $^{5}$ mit Hilfe freiraumplanerischer Prinzipien zum besseren Verständnis des Phänomens „Gemeinschaftsgarten" bei.

In einem zweijährigen Partizipationsprozess entfaltete sich das top-down implementierte Projekt schrittweise $\mathrm{zu}$ einem selbstverwalteten Gemeinschaftsgarten. Dieser Prozess vollzog sich in einer von den Initiator*innen angelegten baulich-räumlichen, sozialen und ökonomischen Rahmenstruktur. Es zeigte sich, dass es weniger relevant ist, wer die Initiative für ein Gartenprojekt setzt (Fachleute oder Bewohner*innen) als die Haltung der Initiator*innen. Sie bringen die Qualität des Anfangens ein, die nach Arendt (1969) untrennbar mit der Qualität des Ausführens verknüpft ist. Dass die Initiator ${ }^{*}$ innen darin nicht verharrt sind und die Entscheidungsmacht vom Projektteam zu den Gärtner*innen verlagert wurde, war eine entscheidende Komponente des Projekts.

Der Gemeinschaftsgarten zeigt, wie über das alltägliche produktive Tätigsein das funktionalistische Abstandgrün in ein gemeinschaftliches Außenhaus (Hülbusch 1978) transformiert werden kann. Alle drei Tätigkeiten einer vita activa - Arbeiten, Herstellen und Handeln - werden im Garten ausgeführt. Im Vordergrund stehen die sowohl individuell als auch gemeinschaftlich ausgeführten (re-)produktiven Tätigkeiten des Gärtnerns (Arbeiten). Mit der im Garten seltener ausgeführten Tätigkeit des Herstellens schaffen die Gärtner*innen hier Dinge, die mit dem Arbeiten in

5 Marit Rosol (2006) unterscheidet in ihrer Dissertation drei Typen von Gemeinschaftsgärten: Nachbarschaftsgärten mit lokalem Bezug, Themengärten wie etwa eine City Farm und thematische Nachbarschaftsgärten.
Verbindung stehen. Über das gemeinschaftliche Organisieren des Gartens und der Gruppe, durch Sprechen und Handeln, schaffen sie zu bestimmten Zeiten einen space of appearance (Arendt 2013). In diesem Erscheinungsraum können die Beteiligten sich selbst in ihrer einzigartigen Individualität und Pluralität erleben und schaffen neue Handlungsspielräume.

Nach einer Einführung in den Kontext des Wiener Gemeindebaus und einer kurzen Geschichte des Gärtnerns in Wien folgt eine Einschätzung der aktuellen Situation der Wiener Gemeinschaftsgärten. Die Darstellung der Ergebnisse beginnt mit einer kurzen Beschreibung der Fallstudie, gefolgt von der Analyse des partizipativen Prozesses vor dem Hintergrund des Konzepts Ladder of Citizen Participation (Arnstein 1969) und der baulich-räumlichen, sozialen und ökonomischen Rahmenstruktur in Bezug auf das Planungsprinzip Innenhaus und Außenhaus (Hülbusch 1978). Das Kapitel schließt mit einer Reflexion der Auswirkungen und weiteren Folgen des Pilotprojekts. Im letzten Teil des Textes werden die Prozesse aus dem Blickwinkel von Arendts Theorie der vita activa und dem dazu im Gegensatz stehenden Leitbild des funktionellen Geschoßwohnungsbaus diskutiert. Der Artikel kommt zum Schluss, dass gerade das Abstandsgrün heute günstige Voraussetzungen für die Umsetzung von Gemeinschaftsgärten im Sinne einer vita activa bietet und dass Gemeinschaftsgärtnern im sozialen Wohnbau Inklusivität fördern kann.

\section{Der zyklische Forschungsverlauf}

Das Pilotprojekt wurde von 2009 bis 2011 nach dem Ansatz der Aktionsforschung (McNiff 2013) von einem Team aus vier Landschaftsplanerinnen und einer Soziologin durchgeführt und 2010 sowohl intern als auch extern evaluiert (Emprechtinger et al. 2010). Die Methode korrespondiert mit dem Ziel, dass externe Initiator*innen und lokale Akteur*innen gemeinsam lernen, wie selbstorganisierte Gemeinschaftsgärten im Kontext des sozialen Wohnbaus umgesetzt werden können. Auch die kritische Reflexion der eigenen Rolle als Initiator*innen, der Einfluss auf den Prozess und die Frage, wer von wem Macht zugesprochen bekommt, wurden dabei angesprochen. Mit dem zyklischen Forschungsverlauf des Planens, Durchführens, Untersuchens und Reflektierens konnte die Theorie der Freiraumplanung Schritt für Schritt in die Praxis umgesetzt und die Praxis eine lebendige Form der Theorie werden (Whitehead 1989). Forschung und Umsetzung 
beruhten dabei auf vorgeleisteten Planungsprinzipien und einigen nicht verhandelbaren Werthaltungen der Initiator*innen wie Gleichwertigkeit, gegenseitiger Respekt, Vertrauen und Fürsorge. Der Prozess war partizipativ, teilweise experimentell und offen, um Neues lernen $\mathrm{zu}$ können (Drummond/Themessl-Huber 2007).

Auch die Erhebung und Analyse aktueller Daten (2018) zur Ergänzung der Fallstudie folgten dem Paradigma qualitativer Forschung und waren durch zirkuläres Vorgehen und Offenheit gekennzeichnet (Lamnek/ Krell 2010). Die dabei durchgeführte Funktions- und Sozialraumanalyse beruht auf einer Interpretation vorhandener soziodemografischer Daten, einer freiraumplanerischen Kartierung des Gemeinschaftsgartens und der Wohnhausanlage, leitfadengestützten Interviews und einer Gruppendiskussion. Zwei Drittel der 27 Gärtner*innen konnten auf diese Weise eingebunden werden. Außerdem stützt sich der Artikel auf Erfahrungen, die von der Autorin über einen Zeitraum von neun Jahren in zahlreichen teilnehmenden Beobachtungssituationen und persönlichen Gesprächen gesammelt wurden. Die Analyse erfolgte mittels methodischer Triangulation (Flick 2011) zur Gegenprüfung der vorliegenden Daten. Die darauf aufbauenden theoretischen Überlegungen zum Thema gemeinschaftliches Außenhaus und gemeinschaftliches Tätigsein sind Gegenstand dieses Artikels und sollen auf diese Weise auch an die am Prozess Beteiligten weitergegeben und allgemein zur Diskussion gestellt werden.

\section{Vom tätigen Leben im Außenhaus}

In ihrem Werk Vita activa oder Vom tätigen Leben unterscheidet Hannah Arendt (1967/1969) drei Grundtätigkeiten einer vita activa, eines tätigen Lebens: Arbeiten, Herstellen und Handeln. Diese drei Tätigkeiten vollziehen die Menschen, um mit ihren Lebensbedingungen zurecht zu kommen. Sie sind nach Arendts Sicht aber auch untrennbar mit einem guten Leben verbunden. Der Begriff vita activa bezeichnet einen im Mittelalter ins Lateinische übersetzten und dabei umgedeuteten Begriff der aristotelischen Lehre für das tätige, politische und von Unruhe gekennzeichnete Leben, das im Gegensatz zur damals höher geschätzten vita contemplativa, dem betrachtenden, beschaulichen Leben steht. Bei Arendt stehen die beiden Lebensweisen gleichwertig auf derselben Ebene nebeneinander.

Mit Arbeiten bezeichnet Arendt alle Tätigkeiten, die mit den biologischen Prozessen des menschli- chen Körpers verbunden und lebensnotwendig sind. Herstellen hingegen bezieht sich auf die Herstellung einer künstlichen Welt von Dingen, die Bestand und Dauer aufweisen und nicht einfach in den lebendigen Prozessen „zerrieben“ werden. Handeln umfasst nach Arendt schließlich die über das Sprechen vermittelten Tätigkeiten, die sich direkt zwischen Menschen abspielen. Handeln ist die politische Tätigkeit schlechthin und dient unter anderem der Begründung und der Erhaltung des Gemeinwesens. Über das Sprechen und Handeln schaffen Menschen einen temporären space of appearance (Arendt 2013), einen Erscheinungsraum, in dem sich die Beteiligten in ihrer einzigartigen Individualität erleben und als Handelnde begreifen können.

Arendt selbst bezeichnete die Unterscheidung der drei Grundtätigkeiten als ungewöhnlich und in der Literatur wenig präsent. Sie berief sich jedoch auf die in den meisten europäischen Sprachen (tote und lebendige) völlig eigenständigen Worte für Arbeiten und Herstellen (labor - work; arbeiten - werken), wobei dem Wort für Arbeiten etymologisch die Nebenbedeutung Not und Mühe anhaftet (Arendt 1967/1969).

In der griechischen Polis galt die Befreiung von der als sklavisch angesehenen Arbeit für Lebensnotwendiges als Ziel des Lebens. Arendt zeigt, wie die Verachtung der lebenserhaltenden körperlichen Arbeit vom griechischen Altertum bis heute wirkt (Arendt 1969). Die Mühen und Plagen der Lebensprozesse haben auch im modernen Leben keinen Platz.

Der Wunsch, sich vom Arbeiten zu befreien, spiegelt sich auch im bis heute wirksamen Leitbild der modernen funktionellen Stadt, wie es in den 1930erJahren in der Charta von Athen formuliert wurde (Hilpert 1984). Es ordnet und trennt das städtische Leben räumlich entsprechend der vier Funktionen: Wohnen, Arbeiten, Erholen und Verkehr. Die Funktion Wohnen steht im Zentrum und ist umgeben von parkartigen Erholungsflächen. Mit der Trennung der Funktionen Arbeiten und Wohnen wurde die Wohnung als Gegenpol der Arbeit materiell umgesetzt. Auch in der Wohnung, der kleinsten Einheit der Funktion Wohnen, erhalten Räume eindeutige Funktionszuweisungen (Wohnzimmer, Schlafzimmer, Küche, Bad, Gang) (Hanisch/Widrich 1999). Die Arbeitsorte der Hausarbeit, früher das Zentrum des Hauses und Hofes, werden auf eine rationell organisierte, raumsparende Kleinküche reduziert (Homann et al. 2002) und durch die Trennung von den anderen Räumen unsichtbar gemacht (Miklautz/Lachmayer 1999) oder ausgelagert 
(Kollektivküchen, Konsum vorgefertigter Nahrungsmittel und Dienstleistungen).

Doch es sind, wie all die Jahrhunderte zuvor, die im Haushalt Tätigen (in der Antike Dienerschaft, Sklaven und Frauen - heute vor allem Hausfrauen, Haushälter*innen, Pflege- und Reinigungskräfte), „welche die immer wiederkehrende Notdurft des Lebens stillen und für müheloses Konsumieren sorgen [...]. Was dies ,müßige Hausgesinde $[$ [...] in Wahrheit ,produzierte, war nicht mehr und nicht weniger als die Freiheit ihrer Herren, oder modern gesprochen, die Bedingung der Möglichkeit ihrer ,Produktivität “ (Arendt 1969: 104). Dabei wird sichtbar, wie der Verachtung für die Subsistenzarbeit die Geringschätzung derer folgte, die sie ausüben (müssen) (Bennholdt-Thomsen 2014).

Die Wurzeln der Freiheit liegen nach Arendt jedoch im Anerkennen des Lebensnotwendigen. Das Annehmen der menschlichen Bedingtheit schafft die Grundlage, auf der Freiheit erst entstehen kann. Im Konzept von Innenhaus und Außenhaus werden die lebenserhaltenden Tätigkeiten ernst genommen und in einer Praxis der Raumorganisation, die diesem Konzept folgt, werden angepasste Arbeitsorte dafür bereitgestellt.

Das Planungsprinzip Innenhaus und Außenhaus (Hülbusch 1978) beschreibt einen Lebensort mit einem vielgestaltigen (haus-)wirtschaftlichen Arbeitsplatz im Sinne der Subsistenz nach dem Vorbild vorindustrieller städtischer Bebauungstypen. ${ }^{6}$ Das Innenhaus ist der Arbeitsplatz für die häusliche Produktion im Haus oder in der Wohnung. Dieses Innenhaus ist eng mit einem Außenhaus verbunden und nur in dieser Kombination vollständig. Das Außenhaus ist der nicht umbaute Raum am Haus, bestehend aus Eingängen, Ausgängen, Vorplätzen, Höfen, Gärten, Schuppen, Kellern, Wegen und Straßen. Diese Orte können im Alltag angeeignet werden (Kurowski 2003) und ihre Aneignung ist eine soziale und ökonomische Notwendigkeit (Hülbusch 1978; Böse 1981). Das Wohnen hat in der Wohnung nur seinen Ausgangsort, von dort aus erschließen sich die Menschen über die täglichen Besorgungen und Gelegenheiten auch das Quartier. Die begriffliche Einheit von Innenhaus und Außenhaus soll den produktiven Zusammenhang von Haus und Hof als kulturhistori- scher Einheit als vollständige Wohn- und Arbeitsorte zeigen (Böse 1981). Innenhaus und Außenhaus entstehen erst im Gebrauch. Es sind relationale Räume, die durch alltägliche Tätigkeiten geschaffen werden. Zentral ist dabei eine enge Verknüpfung von „drinnen“ und „draußen“, aber auch zum Quartier. Das Außenhaus verbindet den privaten Rückzugsort mit dem öffentlichen Handlungsraum (Kölzer 2003). Ein „Draußen“ wird zum Außenhaus, wenn Zuständigkeiten (Verfügungsmacht und deutlich lesbare Grenzen), Zonierungen (Benachbarung verschiedener Nutzungen) und materielle Bedingungen (Nutzbarkeit) gebrauchsorientiert organisiert und grundsätzlich veränderbar sind (Böse 1981).

Am Beispiel des Nachbarschaftsgartens RodaRoda kann der Zusammenhang zwischen dem Innenhaus-Außenhaus-Prinzip von Inge-Meta Hülbusch und Hannah Arendts Theorie einer vita activa verdeutlicht werden. Arendt zufolge finden im privaten Bereich jene ganz persönlichen Tätigkeiten im Sinne von Arbeiten statt (d. h. alle Tätigkeiten, die der Erhaltung des Einzelnen und dem Bestand der Gattung dienen), die verborgen bleiben sollen vor den Augen der anderen und nicht für das Licht der Öffentlichkeit bestimmt sind (Arendt 1969). Im privaten Innenhaus und Außenhaus stellt man sich so dar, wie man ist. Im öffentlichen Raum kann man sich dagegen zeigen, wie man sein will (Hülbusch 1978). Das Außenhaus bildet dabei den Raum diesseits der Grenze zwischen privatem Bereich und der Öffentlichkeit. Hier grenzt das Private, zu dem nicht jeder Zutritt hat, an die Öffentlichkeit mit ihrem Gesehen- und Gehörtwerden. Mit dem Innenhaus und Außenhaus, „dem immer begrenzten, dafür aber greifbaren und handhabbaren Stück Welt" (Arendt 1969: 85), können die täglichen Notwendigkeiten bewältigt und die Voraussetzungen geschaffen werden, um am öffentlichen Raum teilzuhaben. Anders formuliert: „Ohne Eigentum, ${ }^{7}$ wie [John] Locke sagte, können wir mit dem Gemeinsamen nichts anfangen, es ist of no use [Hervorhebung der Autorin]" (Arendt 1969: 86). Das eigene kleine Beet, ein vom Innenhaus getrenntes Stück Außenhaus, platziert in bislang kaum genutzten Grünflächen rund um die Wohnbauten, bietet einen Ausgangspunkt dafür, dass der Grünraum nutzbar - of use - und zum Freiraum wird.

$7 \quad$ Arendt (1969) unterscheidet zwischen Eigentum und Besitz. In der griechischen Polis und in der römischen res publica war Eigentum die Voraussetzung für Bürgerrechte und damit für die Teilnahme am öffentlichen Leben. 


\section{Der Wiener Gemeindebau und seine Freiräume}

Im Zusammenhang mit dem Thema Gemeinschaftsgärten ist der Wiener Gemeindebau aus mehreren Gründen von Interesse: Er ist ein einzigartiges historisches Beispiel fairer Wohnbaupolitik und räumlicher Gerechtigkeit (spatial justice) (Frei 1984), er bietet eine Vielzahl an geeigneten Flächen (meist in unmittelbarer Nähe der Wohnungen, in allen Stadtbezirken, viele davon völlig ohne aktuelle Nutzung), er verfügt über eine zentrale Verwaltung, er bietet leistbaren Wohnraum für mittlere und untere Einkommensschichten ${ }^{8}$ und eine im Prinzip auf soziale Gerechtigkeit ausgerichtete Wohnungsbauund Belegungspolitik (Reinprecht 2007).

Das Wohnbauprogramm des „Roten Wien“ war eine international herausragende sozialpolitische Maßnahme gegen die enorme Wohnungsnot und die kapitalistischen Verhältnisse des damaligen Wohnungsmarktes. Zwischen 1919 und 1934 errichtete die Gemeinde mehr als 65.00o Sozialwohnungen in allen Stadtteilen Wiens (Frei 1984), was zusätzlich einen langfristigen AntiSegregationseffekt zur Folge hatte (Reinprecht 2007).

$\mathrm{Ab}$ 1926, mit dem Bau der ersten sogenannten Superblocks, ${ }^{9}$ folgte der Wiener Gemeindebau verstärkt dem Leitbild des Funktionalismus. Dieser Städtebau der Moderne (Hilpert 1984), der auf das Elend der Arbeitermassen in gründerzeitlichen Stadtquartieren antwortete, leitete mit der räumlichen Trennung der funktionalen Bereiche Arbeiten, Wohnen, Erholen und Bewegen eine radikale Umgestaltung der europäischen Städte ein (Hilpert 1978). Die Folgen dieses planerischen Leitbilds sind in Wien und in vielen europäischen Großstädten bis heute wirksam: große monofunktionale Gewerbegebiete und Wohnhausanlagen, verschwenderische Abstandsgrünflächen mit sehr geringem Gebrauchswert, lange Wege, hohes Verkehrsaufkommen, Zerstörung gewachsener Stadtstrukturen und damit einhergehende Sicherheitsprobleme (Jacobs 1993; Bahrdt 2013).

8 Untersuchung zur Bewohner*innenstruktur der Gemeindebauten 2008: Anteil an Haushalten unter der Armutsgrenze: $29 \%$. Sie ist damit im Gemeindebau seit Jahren höher als im städtischen Durchschnitt (17\%) und stieg in den letzten Jahren weiter an (Sora 2008).

9 Als Superblocks werden innerstädtische Großwohnanlagen mit über 1.0oo Wohnungen bezeichnet. Meist waren diese Anlagen mit integrierten, kollektiv nutzbaren Wohnfolgeeinrichtungen wie Zentralwaschhäusern, Kindergärten, Mutterberatungsstellen, Volksbibliotheken, Veranstaltungs- und Versammlungssälen, Werkstätten und Geschäftslokalen der Konsumgenossenschaft ausgestattet. http://www.dasrotewien.at [09.09.2019].
Das Zentrum des funktionalistischen Leitbilds bilden die sogenannten Wohnmaschinen (Hilpert 1978) mit standardisierten Wohnungen in großer Zahl, umgeben von weitläufigen, parkähnlichen Freiflächen. Die lebenserhaltenden Tätigkeiten der (Re-)Produktion wurden in diesem städtebaulichen Leitbild durch Dienstleistungen ersetzt, aus alltäglichen (Subsistenz-) Arbeitsorten wurde ein gartenarchitektonisch gestaltetes Wohnumfeld. Zur Kompensation der reduzierten Hausarbeitsplätze verfügten die Gemeindebausiedlungen des „Roten Wien“ über zahlreiche kollektive, selbstverwaltete Einrichtungen, auch solche für Hauswirtschaft und Familienarbeit. Diese sollten die Gemeinschaft stärken und die Hausarbeit kompakt organisieren. Gärten waren jedoch ausdrücklich nicht vorgesehen. Dahinter stand eine links-utopistische Haltung und städtebauliche Debatte über „sanitäres“ und „dekoratives“ Grün (Wagner 1915) für die neue, selbstbewusste Arbeiterklasse. Die neuen „Wohnpaläste“ wurden mit repräsentativen Zwecken dienenden Landschaften geschmückt (Homann et al. 2002). Die Arbeiter und ihre Hausfrauen sollten nicht mehr auf bäuerliche Strukturen zur Sicherung ihres Lebensunterhaltes angewiesen sein. Alle gebäudebezogenen Freiflächen waren öffentlich zugänglich. Eine selbstständige Gestaltung und Bewirtschaftung der Außenräume durch die Bewohner*innen wurde damals ebenso wie heute von der Verwaltung der Wohnungen untersagt.

Der Wiener Gemeindebau durchläuft seit einigen Jahren eine Transformation der über viele Jahre sehr stabilen sozialräumlichen Situation. Diese ist auch in den Wohnhausanlagen der Stadterweiterungsgebiete der 196oer- und 7oer-Jahre - wo das Pilotprojekt stattfand - sichtbar. Der soziale Mittelstand zieht weiter ins Stadtumland mit Haus und Garten oder bezieht Wohnungen mit Kaufoption des gemeinnützigen oder frei finanzierten Privatsektors. Mit der 2006 vorgenommenen Änderung der Zulassungsbedingungen zum Gemeindebau trifft eine neu zuziehende Bevölkerung ausländischer Herkunft auf eine alternde, seit langem ansässige Wohnbevölkerung mit stabilen Verhaltenserwartungen (Reinprecht et al. 2011). Um den daraus resultierenden Hierarchien und Konflikten zu begegnen, wird unter anderem die Errichtung von Gemeinschaftsgärten als neuen Aneignungsorten und Investitionen in einen konstruktiven Umgang mit bestehenden Konflikten empfohlen (Reinprecht et al. 2011). Tatsächlich können Gemeinschaftsgärten wertvolle Anknüpfungspunkte für eine bessere Integration sein (Nettle 2016; Müller 2007), damit sie jedoch ihr volles Potenzial entfalten und lang- 
fristig bestehen können, braucht es ein tiefergehendes Verständnis des Phänomens „Gärtnern in der Stadt“.

\section{Die historischen Wurzeln der \\ Gemeinschaftsgärten in Wien}

Nutzgärten zum Anbau von Lebensmitteln waren jahrhundertelang ein wichtiger Bestandteil der Wiener Stadtlandschaft (Auböck 1975). Meist waren es hauszugehörige Nutzflächen, auf denen Obst und Gemüse für den Eigenverbrauch angebaut wurden (Farkas 1999). Erst zu Beginn der Gründerzeit (ab ca. 1850) wurde der städtische hausbezogene Gartenbau von der mittelalterlichen Innenstadt in die sich verdichtenden Vororte verlagert, wo er nach wie vor einen erheblichen Teil der lokalen Nahrungsmittelversorgung abdeckte (Opll/ Csendes 2013). Industrialisierung und Urbanisierung brachten weitere tiefgreifende Veränderungen bezüglich Lebensstilen und Wohnformen, mit massiven Folgen für die innerstädtischen Gemüsegärten. Sie wurden zunehmend als Kontrast zum modernen urbanen Leben betrachtet und durch innerstädtische Freizeiteinrichtungen wie Parks und öffentliche Grünflächen ersetzt (Farkas 1999). An den Rändern der wachsenden Städte entstanden Ende des 19. Jahrhunderts die ersten Kleingärten.

Nach dem Ersten Weltkrieg erreichten die Kleingärten in Wien aufgrund der hohen Arbeitslosigkeit und Lebensmittelknappheit eine erste große Verbreitung. Bürger*innen besetzten freie Flächen in der Stadt, um selbst Nahrungsmittel zu produzieren und die Not zu lindern (Krasny 2012). In Wien sah sich die Stadtregierung letztlich dazu gezwungen, rund 60.000 illegal errichtete Kleingärten zu legalisieren, die Gärtner*innen technisch zu unterstützen und vor Wucher und Kündigung zu schützen (Neurath 1923; Förster 2006).

Im Wiener Gemeinderat gab es nach dem Ersten Weltkrieg zwei Strategien zur Bewältigung der enormen Wohnungsnot in Wien: die Siedlerbewegung und den kommunalen Wohnbau. Die Siedlerbewegung war von bürgerlichen Strukturen unabhängig, genossenschaftlich organisiert und auf Subsistenz ausgerichtet (Blau 1999). Im Gegensatz zu den vom Wohnort getrennten Kleingärten waren die Siedlerparzellen ein „ganzes Haus“ mit allen für die Selbstversorgung erforderlichen Elementen, inklusive der Gemeinschaft der Siedler*innen. Um das „wilde“ Siedeln in geordnete Bahnen zu lenken wurde ein Siedlungsamt in der städtischen Administration eingerichtet (Krasny 2012).
Auch während des Zweiten Weltkriegs fand in Wien eine massive informelle Landbesetzung mit Tausenden von Gemüsegärten statt, die das Überleben der Stadtbewohner*innen sichern sollten (Novy/ Förster 1985; Krasny 2012). Gartenhütten wurden zu stabilen Häusern ausgebaut und nach dem Krieg von der Stadtverwaltung legalisiert (Appel et al. 2011). In den folgenden Jahrzehnten des wachsenden wirtschaftlichen Wohlstandes (196oer bis 1990er) verloren die Kleingärten für die städtische Lebensmittelproduktion jedoch massiv an Bedeutung. Die Erholungsfunktion der Kleingärten trat in den Vordergrund und eine neue Kategorie in der Flächenwidmungsplanung ermöglichte ab den 1970er-Jahren ihre dauerhafte Umgestaltung in Wohngebiete (Shimpo et al. 2014). Heute sind von den 600 Hektar Kleingärten im Jahr 1953 nur mehr 85 Hektar im annähernd ursprünglichen Zustand erhalten (StadtWien 2018).

Eine andere Wurzel der heutigen Gemeinschaftsgärten ist die vor-industrielle Allmende (Taborsky 2008). In Österreich gab es vom Mittelalter bis um etwa 1800 (in manchen Bereichen auch heute noch) diese Form der Gemeinschaftsnutzung von lokalen, kollektiven Ressourcen. Sie war geprägt von gemeinsamen Nutzungsrechten und gemeinsamer Pflegeverantwortung in relativ geschlossenen sozialen Kleinsystemen von überschaubarer Komplexität (Marquardt 2002). Dieses Organisationsmodell der multifunktionalen Ressourcennutzung ist zwar nicht direkt auf die moderne Gesellschaft übertragbar (Marquardt 2002), Gemeinschaftsgärten weisen jedoch einige Übereinstimmungen mit den historischen Formen der Allmende auf (Taborsky 2008).

Diese historische Betrachtung zeigt die lange Tradition der städtischen Gärten in Wien und des gemeinschaftlichen Wirtschaftens in Österreich, von der auch die neuen Formen des gemeinschaftlichen Gärtnerns beeinflusst sind.

\section{Die aktuellen Entwicklungen gemeinschaftlichen Gärtnerns in Wien}

Seit $2008^{10}$ hat sich in Wien mit den Gemeinschaftsgärten eine neue Gartenbewegung entwickelt. Sie wird sowohl von der bottom-up community garden-Bewegung der 1970er-Jahre in New York (Follmann/Viehoff

10 Einige wenige Initiativen gab es bereits früher (z.B. Yppengarten https://yppengarten.wordpress.com/ [29.11.2019] und Boku-Gemeinschaftsgarten http://www. boku-gemeinschaftsgarten.org/ [20.11.2019]. 
2014) als auch von dem sehr erfolgreichen, stärker topdown geprägten deutschen Modell der Interkulturellen Gärten (Müller 2007) beeinflusst.

Die neuen urbanen Garteninitiativen in Wien sind äußerst heterogen. Sie unterscheiden sich hinsichtlich Größe (500 bis $7.500 \mathrm{~m} 2$ ), Lage (innerstädtisch - Stadtrand) sowie gärtnerischer (Hochbeete bis Grabeland), rechtlicher (Einzelpersonen, Vereine, ohne Titel) und organisatorischer Struktur (selbstorganisiert, begleitet von Organisationen, betreutes Gärtnern). Gemeinsam ist ihnen, dass sie deutlich mehr Aneignungsmöglichkeiten und Selbstbestimmung ermöglichen als öffentliche Parkanlagen (Rosol 2006).

Eine aktuelle Publikation über Gemeinschaftsgärten in Wien (Exner/Schützenberger 2018) unterscheidet zwei grundsätzliche Typen bezüglich ihrer Governance-Struktur: zum einen selbstorganisierte Gärten (grassroots community gardens) und zum anderen solche, die von der Stadt ${ }^{11}$ initiiert und betreut werden (municipally-influenced community gardens). Etwa zwei Drittel der untersuchten Gärten werden von der Stadt verwaltet. Die Autor*innen charakterisieren die bestehenden Gemeinschaftsgärten in Wien als post-fordistische Stadträume, die sich durch einen projektähnlichen Charakter, informelle Netzwerke und geringe rechtliche und finanzielle Sicherheit auszeichnen. Bei den Gärtner*innen handelt es sich überwiegend um Personen mit großem sozialem, kulturellem und z.T. wirtschaftlichem Kapital, während Personen mit niedrigen Bildungsabschlüssen und sozial benachteiligte Gruppen stark unterrepräsentiert sind.

Rund 12 \% der Menschen in Österreich sind laut einer Studie der Europäischen Kommission zur Bekämpfung von Armut und sozialer Ausgrenzung armutsgefährdet, ${ }^{12} 6 \%$ gelten als „manifest arm“ (TillTentschert et al. 2011). Im sozialen Wohnbau liegt der

11 Die meisten davon wurden von der Gebietsbetreuung oder der Lokalen Agenda 21 initiiert und weiter verwaltet.

12 Die aktuelle einkommensspezifische Armutsgefährdungsschwelle für einen Einpersonenhaushalt beträgt laut EU-SILC $2018951 €$ pro Monat (http://www.statistik. at/web_de/frageboegen/private_haushalte/eu_silc/index. html [29.11.2019]. In Österreich sind 12,4 \% der Bevölkerung armutsgefährdet (Stand 2009). Hochgerechnet auf die Gesamtbevölkerung liegt die Zahl der armutsgefährdeten Personen zwischen rund 940.000 und 1,1 Millionen. Das höchste Armutsrisiko besteht für Personen mit ausländischer Staatsbürgerschaft. Ebenfalls stark armutsgefährdet sind Personen in Ein-Eltern-Haushalten (STATISTIK AUSTRIA 2009).
Anteil der Haushalte unter der Armutsgrenze mit $29 \%$ im Vergleich zu 17 \% deutlich höher als in der Gesamtbevölkerung (Sora 2008). Auch der Anteil an Personen mit Pflichtschulabschluss als höchster Qualifikation (25 $\% \mathrm{zu} 17 \%$ ) und der Anteil an (Fach-)Arbeiter*innen, Angelernten und Hilfsarbeiter*innen ist im Wiener Gemeindebau deutlich höher als im Wiener Durchschnitt. Die beiden letzten Gruppen machen ein Viertel der Gemeindebau-Bewohner*innen aus. Auch der zur Verfügung stehende Wohnraum pro Person ist mit 30 $\mathrm{m}^{2}$ gegenüber $40 \mathrm{~m} 2$ signifikant geringer als im städtischen Durchschnitt (Sora 2008).

Bei diesen Problemlagen setzte das Pilotprojekt Nachbarschaftsgarten Roda-Roda an und entwickelte eine Umsetzungsstrategie für Gemeinschaftsgärten für diesen Kontext.

\section{Das Pilotprojekt Nachbarschaftsgarten Roda- Roda}

Der Nachbarschaftsgarten Roda-Roda befindet sich am nördlichen Stadtrand von Wien. Der Bezirk Floridsdorf ist ein Stadterweiterungsgebiet, baulich-räumlich geprägt von einer Mischung aus sozialen Wohnhausanlagen der 196oer-Jahre, Einfamilienhäusern und Gewerbebetrieben.

Der Garten liegt am Rand der kommunalen Wohnhausanlage Oskar-Helmer-Hof. Die Wohnanlage ist eine typische Stadtrandsiedlung der 196oerJahre und ein charakteristisches Beispiel für den funktionalistischen Städtebau der Nachkriegszeit, errichtet aus Betonfertigteilen auf einem ehemaligen Militärgelände (Czeike 2004). Der Komplex besteht aus 30 frei stehenden Zeilenbauten mit zwei bis vier Etagen und insgesamt 788 Wohnungen, umgeben von verkehrsberuhigten, landschaftsparkartigen Grünflächen (Rasenflächen, Strauchgruppen, Bäume). Es gibt mehrere Gemeinschaftseinrichtungen (öffentlicher Kindergarten, öffentliche Schule, öffentliches Kinderfreibad, Ballspielbereiche, Spielplätze), einen Nahversorger und ein Café. Die Gestaltung des Wohnumfeldes folgte dem städtebaulichen Leitbild Sanitäres Grün mit Licht, Luft und Sonne für alle Wohnungen und ist ein Resultat der damals neuen Montagebauweise und den dafür notwendigen Abständen zwischen den Gebäuden (Marchart 1984). Die Nutzung ist damals wie heute durch die Funktionszuweisung als Erholungsraum sowie durch die Ausstattung, die Bepflanzung, die Hausordnung und die hohe soziale Kontrolle stark eingeschränkt. 


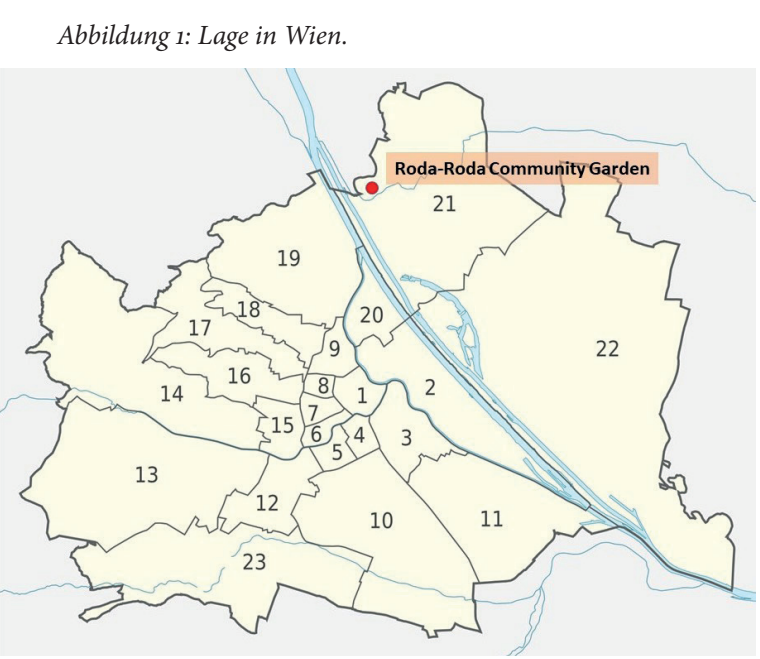

(Grundlage: stadtplanwien36o.at)

Abbildung 3: Abstandsgrün Oskar-Helmer-Hof 2008.

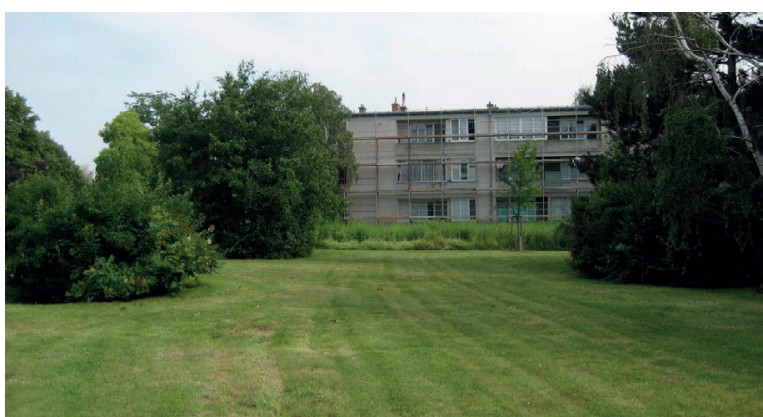

CC BY-NC-SA Rita Mayrhofer

Ein interdisziplinäres Projektteam aus vier Landschaftsplanerinnen und einer Soziologin, dem die Autorin angehörte, startete im Herbst 2008 ein dreijähriges Pilotprojekt zur Erprobung von Gemeinschaftsgärten im Wiener Gemeindebau. Im ersten Jahr (2009) stellte das Projektteam die notwendigen Rahmenbedingungen (räumlich, sozial, wirtschaftlich und legistisch) her und übertrug in den zwei folgenden Jahren alle Aufgaben und Verantwortlichkeiten schrittweise an die Gärtner*innen. Frauen wurden dabei gezielt unterstützt, in den formal geforderten hierarchischen Governance-Strukturen (Verein) eine ihrer Zahl und ihrem Engagement angemessene Rolle einzunehmen.

Das Projekt bestand aus zwei eng ineinandergreifenden Prozessen: dem Planungsprozess zur Herstellung einer tragfähigen Rahmenstruktur und dem Partizipationsprozess zur Umsetzung der Selbstorganisation. In den folgenden Kapiteln werden die beiden Prozesse beschrieben und ihre Wirkungen analysiert. Dazu wird der Partizipationsprozess anhand der
Abbildung 2: Lage im Oskar-Helmer-Hof

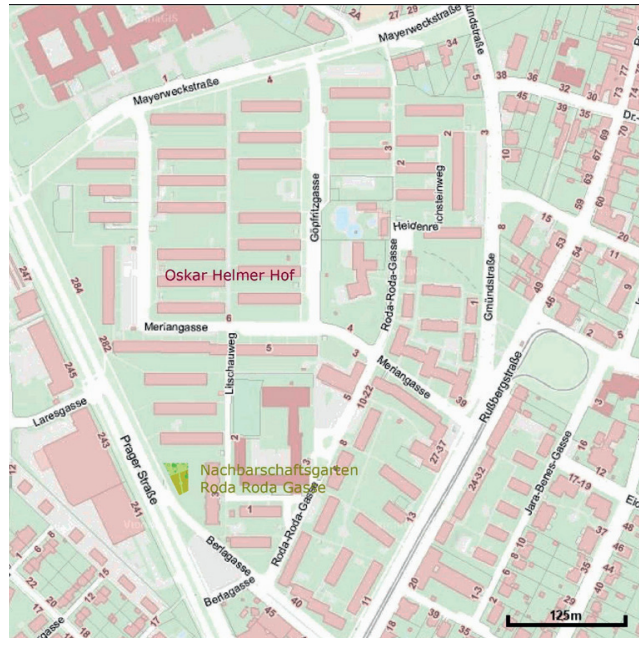

(Grundlage: wien.at: Magistrat der Stadt Wien)

Abbildung 4: Nachbarschaftsgarten Roda-Roda 2009

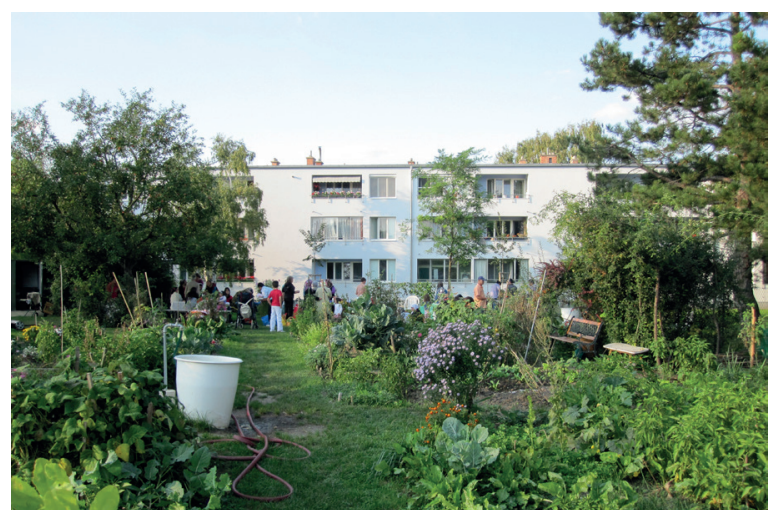

CC BY-NC-SA Rita Mayrhofer

Ladder of Citizen Participation (Arnstein 1969) und die Rahmenstruktur des Gartens anhand des Planungsprinzips Innenhaus und Außenhaus diskutiert.

\subsection{Der Partizipationsprozess als schrittweise Umverteilung der Macht}

Die Gärtner*innen sind in das Gartenprojekt an einem Punkt eingestiegen, an dem das Projektteam eine klare Führungsposition einnahm und die Rahmenstruktur des Gartens (Ort, Größe, Begrenzung und Zonierung) bereits festgelegt war. Dies nahm einerseits Entscheidungen vorweg, ersparte den Gärtner*innen aber andererseits die potenziell aufreibenden ersten Schritte des Realisierungsprozesses. Die Beteiligten konnten sicher sein, dass die Teilnahme in der ersten Phase keine unvorhersehbaren Risiken birgt.

Der Partizipationsprozess bestand aus mehreren weitgehend aufeinanderfolgenden Phasen, die Schritt 
Abbildung 5: Zeitliche Entwicklung des Beteiligungsprozesses anhand des Konzepts Ladder of Citizen Participation von Sherry Arnstein

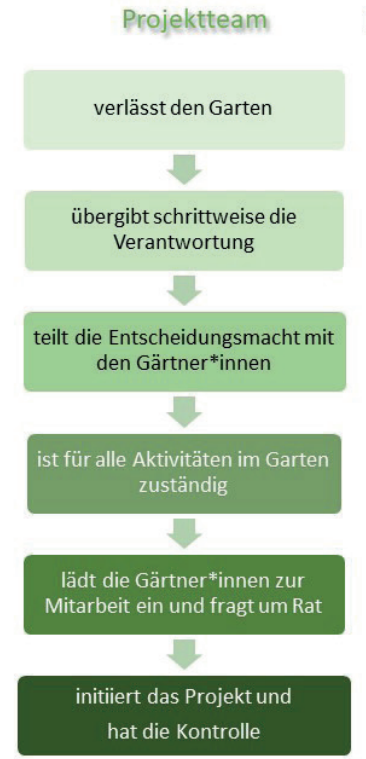

Ladder of participation

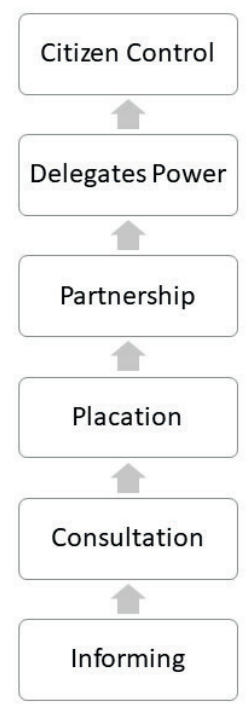

CC BY-NC-SA Rita Mayrhofer 2018
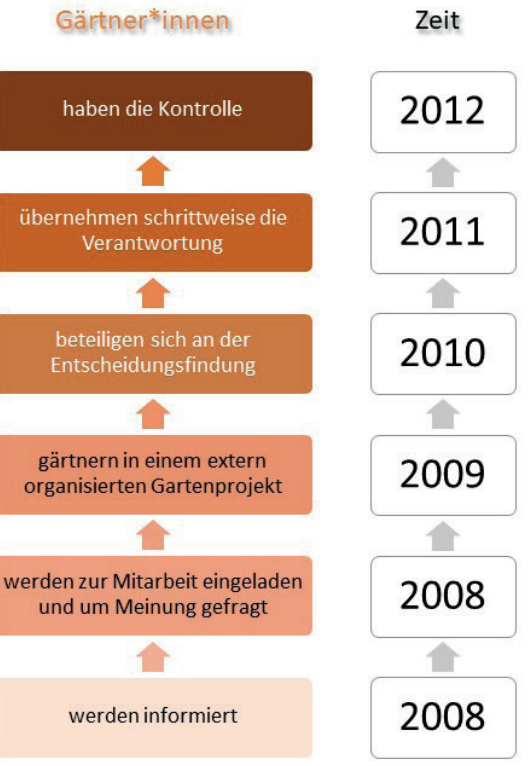

Partnerschaft, delegierte Entscheidungen und zuletzt Übergabe der Entscheidungsmacht) kann innerhalb eines Prozesses eine zunehmende Ermächtigung der Bürger*innen erfolgen. Die Fallstudie zeigt, wie der Partizipationsprozess als zueinander gegenläufige Bewegungen auf der Leiter gesehen werden kann, bei dem das Projektteam und die Gärtner*innen im Verlauf der Umverteilung der Macht die Balance halten.

Das Projektteam startete 2008 von einer Position der weitgehenden Entscheidungsmacht und reduzierte schrittweise Input und Kontrolle bis zum Ausstieg aus dem Projekt. Die meisten Gärtner*innen waren anfänglich primär daran interessiert, in unmittelbarer Nähe ihrer Wohnung eigene Pflanzen (Gemüse, Obst, Blumen) anzubauen und Zeit im Freien zu verbringen. Der Prozess verlief parallel zu den wachsenden Fähigkeiten der Gärtner*innen und der sich entwickelnden Gartengemeinschaft. Die Gärtner*innen stiegen schrittweise von der Stufe des informing (Einladung zum Tätigwerden) bis zur citizen control (Übernahme aller Agenden und der Verantwortung). Mit der zunehmenden Erfahrung aller Beteiligten wuchs auch das Vertrauen in das Verfahren zur kooperativen Entscheidungsfindung und in die gemeinsam getragene Verantwortung (Mayrhofer 2018). Hier unterscheidet sich das Projekt von vielen anderen extern initiierten Gemeinschaftsgärten in Wien, wo die Initiator ${ }^{*}$ innen die Letztverantwortung und damit auch die Kontrolle dauerhaft behalten. den Schritten (Information, Konsultation, Beratung, 
Das Modell der Leiter verbessert das Verständnis des Prozesses, es hat allerdings auch Grenzen. Die Gärtner*innen sind beispielsweise keine homogene Gruppe. Innerhalb der Gartengemeinschaft gibt es verschieden strukturierte Machtgefälle. Die Verlagerung der Macht von den Initiator ${ }^{\star}$ innen fand nicht auf alle Gärtner*innen gleichermaßen statt. Manche Personen können oder wollen sich nicht in die Entscheidungsfindung einbringen. So hat vorwiegend der Vereinsvorstand die Kontrolle und die Verantwortung für die selbstbestimmte Gartengemeinschaft übernommen. Er besteht aus vier bis sechs Personen und wird alle zwei Jahre gewählt. Im Beobachtungszeitraum waren überwiegend Frauen mit österreichischer Muttersprache und ohne Doppel- und Mehrfachbelastung im Vorstand aktiv. Auch bezüglich der Reichweite der Entscheidungsmacht besteht keine vollkommene Kontrolle. Die Gärtner*innen können innerhalb des Gartens zwar autonom entscheiden, nach außen sind sie an die Verträge mit Wiener Wohnen gebunden.

Der Entstehungsprozess des Nachbarschaftsgartens Roda-Roda kann neben der Ladder of Citizen Participation auch über eine zeitliche Abfolge verschiedener Governance-Modelle beschrieben und verstanden werden (McGlone 1999; Nettle 2016; Exner/Schützenberger 2018; Fox-Kämper et al. 2017). Ein Gemeinschaftsgarten ist urban governance auf lokaler Ebene. Der Gegenstand des gemeinsamen Handelns ist eine Gemeinschaftsaufgabe, etwas, das durch Einzelne nicht erreicht werden kann. Hier werden gemeinsame Situationsdeutungen, Ziele und Maßnahmen ausgehandelt und neue Netzwerke geknüpft, die neben den bestehenden traditionellen Government-Strukturen bestehen (Fürst 2007). Das Projekt startete mit einem Top-down-Konzept (von Fachleuten ohne anfängliche Vertretung der Gärtner*innen entwickelt), wurde durch den Beteiligungsprozess zu einem Prozess des bottomup with professional help, dann einem bottom-up with informal help und hat sich letztendlich zu einer Bottomup-Governance-Struktur entwickelt (lokale Gartengemeinschaft leitet und organisiert sich selbstständig) ${ }^{13}$.

13 Fox-Kämper et al. (2017) identifizierten auf Basis einer Literaturrecherche von 38 Publikationen eine Typologie von Governance- Strukturen: (1) top-down, (2) top-down with community help, (3) bottom-up with political and/or administrator support (PAS), (4) bottom-up with professional help, (5) bottom-up with informal help, (6) bottom-up. Sie beschreiben auch gemischte Formen und unterscheiden drei verschiedene Entwicklungsstadien: planning/design, construction/implementation und management.
Die Abfolge der Governance-Modelle zeigt die verschiedenen Stadien der Selbstorganisation und wie das Zusammenspiel von politisch-administrativen und zivilgesellschaftlichen Akteur*innen (Wohnhausverwaltung und Bewohner*innen) mit unterschiedlichen Maßnahmen gefördert werden kann.

Das Pilotprojekt verdeutlicht, dass es weniger um eine bestimmte Technik der Partizipation als um das Bewusstsein der „Machthaber*innen“ (im vorliegenden Fall der Initiator*innen) für die bestehenden Machtverhältnisse geht und darum, wie Partizipationsprozesse ungleich verteilte Einflussmöglichkeiten aufrechterhalten oder verändern können (Stout 2010).

Die Initiative für das Gartenprojekt ging nicht von den Gärtner*innen selbst aus, sondern von Fachfrauen, die die Chance und die Potenziale erkennen und ausschöpfen konnten. Die Bewohner*innen selbst konnten und wollten diese initiativen Schritte aufgrund verschiedener Hindernisse nicht setzen. Als die Idee des Gemeinschaftsgartens Form annahm und sie in einem sicheren Rahmen eingeladen wurden aktiv zu werden, haben sich 24 Personen bzw. Familien beteiligt und den Garten gemeinsam mit den Initiator*innen umgesetzt. Beide Aktionen - die Initiative und das Umsetzen - entsprechen dem ursprünglichen Sinn des Wortes Handeln.

In der griechischen und lateinischen Sprache gab es für das, was wir heute als Handeln bezeichnen, zwei Worte, die zwei klar unterscheidbare Stadien des Handelns benennen: das Anfangen/Anführen (agere) und das Ausführen/Betreiben (gerere). Arendt zeigt, wie aus den eng verbundenen Bezeichnungen zwei getrennte Funktionen wurden: das Befehlen und das Befehle ausführen. Beide bleiben in wechselseitiger Abhängigkeit. Doch wird aus dem ursprünglichen Bezug zwischen dem Einen, der allein anfängt, und den Vielen, die gemeinsam vollbringen, das Befehlen und Vollstrecken, und zwar dann, wenn aus dem Anfänger und Anführer ein Herrscher wird, der sich dauerhaft in der Position des Anfangenden befindet (Arendt 1969).

Auch Gartenprojekte brauchen Anfänger*innen und Anführer*innen, die die Initiative ergreifen, das Risiko auf sich nehmen und damit jene Kraft entwickeln, die die Stärke der Starken ausmacht (Arendt 1969). Und sie brauchen die Kräfte der Vielen, die das Werk vollbringen und ohne die die Stärke der Anfänger*innen und Anführer*innen machtlos bliebe. Auf dieses Zusammenspiel ist auch eine Gartengemeinschaft angewiesen. 
Abbildung 6: Plan des Gartens, wie er auch derzeit besteht, mit Gemeinschaftsbereich (A) und individuellen Beeten (B)

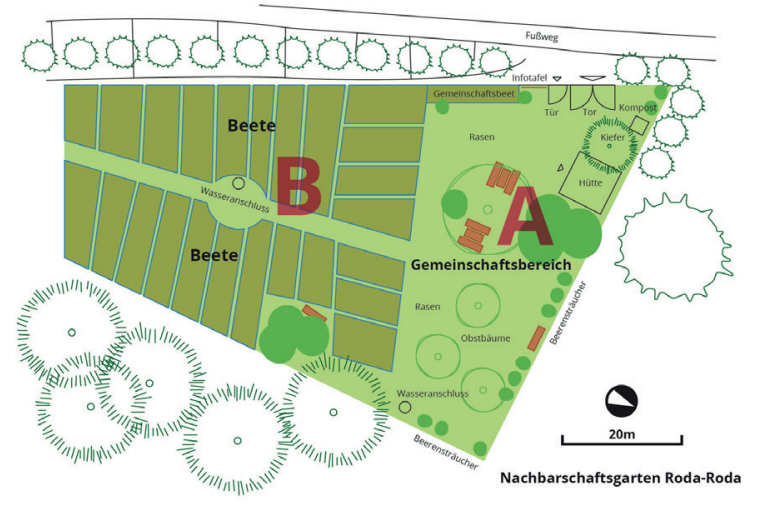

CC BY-NC-SA Rita Mayrhofer 2018

2018 waren 27 Erwachsene im Alter zwischen 30 und 90 Jahren im Garten tätig. Davon waren 18 Frauen und 9 Männer, 11 davon sind Gründungsmitglieder. Im Garten wird Deutsch, Türkisch, Philippinisch, Bosnisch und Arabisch gesprochen (gereiht nach Häufigkeit). Nicht alle Gärtner*innen sprechen Deutsch und von den deutschsprachigen Personen spricht niemand eine der anderen Sprachen. Die fehlende gemeinsame Sprache wirkt als Barriere und fördert die Bildung von Subgruppen innerhalb der Gemeinschaft. Auch gegenseitige kulturelle Zuschreibungen, ethnisierte Konflikte und der einseitige Rückzug aus Diskussionen stehen im Zusammenhang mit den Verständigungsproblemen. Die Gärtner*innen betonen, dass sie sich gut verständigen, da grundsätzlich ein angenehmes Gesprächsklima im Garten herrsche. Jene, für die Deutsch eine Fremdsprache ist, unterstreichen, dass die Gespräche im Garten sie dabei unterstützen, mehr Deutsch zu sprechen.

Es gibt keine erzwungene Rotation der Beete. Das stärkt die Stabilität der Gemeinschaft und erleichtert die Integration neuer Personen. Jährlich werden ein bis zwei Beete frei (überwiegend aus persönlichen Gründen wie Geburt, Krankheit, Tod, Jobangebot, Wohnungswechsel), die dann gemäß einer Warteliste vom Vereinsvorstand nachbesetzt werden.

7.2 Die baulich-räumliche, soziale und ökonomische Rahmenstruktur des Gemeinschaftsgartens

Der Nachbarschaftsgarten Roda-Roda ist über einen öffentlichen Fußweg erreichbar. Er hat insgesamt 800 $\mathrm{m} 2$, davon sind $300 \mathrm{~m} 2$ Gemeinschaftsbereich (Fläche
Abbildung 7: Zonierung des Gartens in verschiedene Nutzungsbereiche

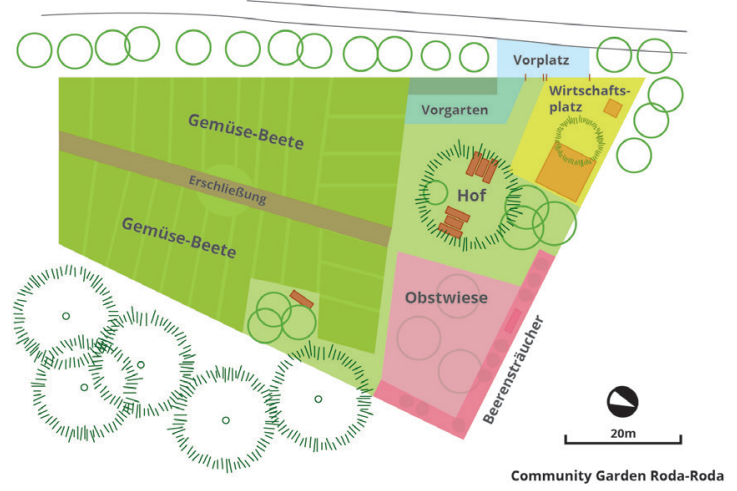

CC BY-NC-SA Rita Mayrhofer 2018

A) und $500 \mathrm{~m} 2$ individuelle Gemüsebeete (Fläche B). Die 24 Beete sind zwischen 9 und $15 \mathrm{~m} 2$ groß und werden individuell bewirtschaftet. Der Garten ist eingezäunt (Zaunhöhe 1,20 m) und nur an bestimmten Tagen für die Öffentlichkeit zugänglich. Im Inneren gibt es keine Zäune, sondern nur niedrige Einfassungen, Wege und Pflanzen zur Abgrenzung der verschiedenen Bereiche.

Der Garten ist in mehrere Nutzungsbereiche gegliedert: Vorplatz - Vorgarten - Wirtschaftsplatz - „Hof“ (Gemeinschaftsbereich mit Sitzplatz) - Obstwiese - Beerensträucher und Blumenbeete - Erschließung - Gemüse-Beete. Diese Abfolge von Räumen ergibt sich aus den Tätigkeiten, die dort stattfinden, und aus dem Verhältnis der Tätigkeiten zueinander.

Diese Zonierung wurde von den Initiator*innen festgelegt und bildet die baulich-räumliche Rahmenstruktur des Gartens. Der Rahmen ermöglicht und verhindert Tätigkeiten und bietet dadurch eine gewisse Handlungssicherheit. Die Grundausstattung bestand zu Beginn nur aus einer Rasenfläche, Sitzgelegenheiten, Erdwegen und einfachen Beeteinfassungen. Die Zonierung und die Ausstattung waren bewusst einfach gestaltet, um eine spätere Ausgestaltung durch die Gärtner*innen zu ermöglichen.

Diese baulich-räumliche Rahmenstruktur beruht auf dem Planungsprinzip Innenhaus und Außenhaus (Hülbusch 1978). Sie orientiert sich zum einen an der Gartenarbeit und ist im Sinne eines Außenhauses organisiert (Zuständigkeiten, Zonierung und Ausstattung). Die direkte Verknüpfung von „drinnen und draußen“ ist zwar nicht gegeben, da der Garten nicht direkt über die einzelnen Wohnungen erreichbar ist, doch ist die Distanz zu den Wohnungen klein genug, um das täg- 
liche Tätigsein und eine Erweiterung des häuslichen Arbeitsplatzes zu ermöglichen. Die Gärtner*innen sind draußen präsent und knüpfen vielfältige Kontakte im Garten und über den Zaun hinweg. Der Garten bietet damit eine gute Verknüpfung von der Wohnung zum Quartier. Zum anderen orientiert sich die Rahmenstruktur am gemeinschaftlichen Tätigsein und erweitert damit das Prinzip Außenhaus um neue Elemente gemeinschaftlicher Organisation und Nutzung. Der Garten ist gemeinsamer Besitz und kein Privateigentum. Der Gebrauchswert und nicht der Tauschwert der Fläche steht im Vordergrund. Die Beete stellen einen privaten Raum im Sinne der Verfügbarkeit dar. Hier können die Gärtner*innen autonom tätig sein und allein entscheiden. Die Beete sind für manche Frauen auch ein Rückzugsort, wo sie in Ruhe einer naturverbundenen Tätigkeit nachgehen und für sich sein können. Der gemeinschaftliche Bereich ist hingegen ein gemeinschaftlicher, öffentlicher Handlungsraum, in dem Absprachen verhandelt und eingefordert werden und in dem die Gärtner*innen Anlässe finden einander zu begegnen.

Die soziale und ökonomische Struktur des Nachbarschaftsgartens Roda-Roda ist auf Selbstorganisation und Subsistenz ausgerichtet. Der von den Gärtner*innen gewählte Vorstand trifft als Team die notwendigen Entscheidungen. Er vertritt alle nach außen und nimmt neue Mitglieder auf. Ein unbefristeter Vertrag mit dem Grundstückseigentümer Wiener Wohnen sichert das längerfristige Bestehen des Gartens. Die finanziellen Mittel für die Errichtung wurden durch das Pilotprojekt und durch Sponsoring erbracht. Die laufenden Kosten sind gering und werden durch einen Jahresmitgliedsbeitrag gedeckt, der für alle Gärtner*innen gleich hoch ist. Zusätzlich betreiben die Gärtner*innen auch Tausch- und Schenkökonomie.

\section{Wirkungen des Nachbarschaftsgartens}

Die Wirkungen, die der Garten für die Gärtnerinnen und in der Wohnhausanlage insgesamt entfaltet hat, sind vielfältig. Viele dieser Wirkungen wurden auch bereits an anderen Orten wissenschaftlich untersucht und dokumentiert. Gemeinschaftsgärten ermöglichen die Eigenproduktion von gesundem Gemüse (Turner 2011; Pudup 2008; Macias 2008), sie verbessern das Wohlbefinden der Gärtner*innen und ihrer Angehörigen (Armstrong 2000; McCormack et al. 2010), sie unterstützen den Aufbau von sozialem und kulturellem Kapital (Glover et al. 2005) und die Interaktion mit anderen sozialen Gruppen und Ethnien (Shinew et al. 2004), sie fördern den Sinn für Gemeinschaft (Ohmer et al. 2009; Macias 2008), ermöglichen individuelles und gemeinsames Lernen (Madlener 2009), verhelfen zu Erfahrungen im Organisieren von Gemeinschaft (Schmelzkopf 2002; Saldivar-Tanaka/Krasny 2004) und bieten Erholung und Naturerleben (Ferris et al. 2001; Kurtz 2001; Taborsky 2008). Alle diese Wirkungen konnten auch im Fallbeispiel nachgewiesen werden.

Die Analyse der Gartenpraxen anhand des Tätigkeitskonzepts von Hannah Arendt nach Arbeiten, Herstellen und Handeln zeigt, dass jede der drei Tätigkeiten in anderen räumlichen Rahmenbedingungen stattfindet und andere Räume bedingt, wie sie sich in der Zonierung des Gartens zeigen. Die Gärtner*innen graben, pflanzen, jäten, gießen, mähen, ernten, zerkleinern, kompostieren, ziehen Samen, teilen die Ernte, bewegen sich, räumen auf, bringen Essen in den Garten, erleben Natur, genießen Sonnenlicht, helfen sich gegenseitig und tauschen Wissen und Erfahrungen aus. Dieses Arbeiten ist im Garten aufgrund der Rahmenstruktur möglich und produziert zugleich durch die körperbezogenen Subsistenztätigkeiten Arbeitsplätze im Garten. Tätigkeiten des Herstellens finden im Fallbeispiel weit seltener statt als das Arbeiten. Tätigkeiten wie Beeteinfassungen bauen, Hütte errichten, Sitzplatz pflastern oder Hochbeete bauen dienen überwiegend dem Gärtnern. Das hängt auch mit dem Fokus der Initiator*innen und der Gärtner*innen auf das Gärtnern im Sinne von Arbeiten und weniger im Sinne von Herstellen zusammen. Über manche Tätigkeiten, vor allem von Männern, wurde sichtbar, dass es ein starkes Bedürfnis nach Herstellen und nach einer Werkstatt gibt, dass der Garten aber nur bedingt der geeignete Ort dafür ist. Das Herstellen von über die eigene Existenz hinausgehenden Dingen wirkt langfristiger, braucht Platz und steht mit dem Nutzungsvertrag in Konflikt. Jedoch haben viele Gärtner*innen über ihre Teilhabe hinaus Spuren im Garten hinterlassen (Bänke, Hochbeete, Kinderspielhäuser, Bewässerungshilfen), die auch heute noch mit ihnen in Zusammenhang gebracht werden und einen Teil der Geschichte des Gartens bilden. Die Tätigkeiten der dritten Kategorie - das Handeln - finden in verschiedenen Formen statt: Ideen einbringen, diskutieren, Probleme besprechen, Abläufe klären, Regeln aushandeln, Regelverletzungen besprechen, neue Lösungen finden, Feste feiern, streiten und verzeihen. Sie dienen der Schaffung und Erhaltung des lokalen Gemeinwesens im Garten. 
Während Arbeiten und Herstellen auch allein getan werden können, ist das Handeln nur vorstellbar in der Gesellschaft der Menschen. Im Sprechen und Handeln werden die Menschen in ihrer Einzigartigkeit erlebbar. Handelnd können sie sich voneinander unterscheiden, statt nur verschieden zu sein. Handeln ist ein aktives In-Erscheinung-Treten, eine Initiative, die Menschen selbst ergreifen, um sich zu zeigen. Im gemeinsamen Handeln entsteht ein Erscheinungsraum, ein space of appearance (Arendt 2013), der nur während der gemeinsamen Tätigkeit besteht und verschwindet, wenn das Handeln beendet ist. Er ist potenziell immer da, wenn Menschen zusammenkommen und gemeinsam ein Machtpotenzial bilden. Macht - hergeleitet von ,mögen und ,möglich' (Arendt 1969) - kann nur entstehen, wenn Menschen miteinander tätig sind und zwar so nahe, dass die Möglichkeit zum Handeln gegeben ist.

Handeln und Sprechen brauchen eine gemeinsame Sprache, und genau darin liegt eine große Herausforderung für Gemeinschaftsgärten mit hoher Diversität. Sprachbarrieren und Verständigungshindernisse blockieren Gärtner*innen dabei, sich am Handeln zu beteiligen und am sich entfaltenden Machtpotenzial teilzuhaben. Hier braucht es ein stetes Bemühen der Beteiligten, die Barrieren zu überwinden, und den Entschluss der Einzelnen, am Handeln teilzunehmen. Nur so können sie sich das Potenzial erschließen und sich selbst in die Welt einschalten (Arendt 1969). Durch die Initiative eines professionellen Teams konnte für manche Menschen der Entschluss des In-ErscheinungTretens in der Welt des Gemeinschaftsgartens erleichtert bzw. gar erst ermöglicht werden.

Der Nachbarschaftsgarten Roda-Roda entfaltete auch Wirkungen in der Wohnhausanlage Oskar-Helmer-Hof. Im gesamten Bereich findet aktuell eine verstärkte gärtnerische Aneignung der Bewohner*innen im Nahbereich der Wohnungen statt. Diese neuen, nicht genehmigten Pflanzungen vor Balkonen und Eingängen sind zwischen einem und fünf Quadratmetern groß. Sie bestehen überwiegend aus blühenden Stauden, Sträuchern, Kräutern, Obst und seltener auch aus Gemüsepflanzen. Gepflanzt und gepflegt werden sie von Menschen, die entweder aus dem Gartenprojekt ausgestiegen sind, oder solchen, die zusätzlichen Platz für ihre Pflanzen suchen oder die durch den Garten inspiriert wurden und sich durch das Gartenprojekt indirekt legitimiert fühlen. Die inzwischen 38 privaten Pflanzungen außerhalb des Pilotprojekts veranlassten die Verwaltung dazu, neue Regeln für die gärtnerischen Aktivitäten ihrer Mieter*innen zu verhandeln.
Der Nachbarschaftsgarten Roda-Roda erlangte eine gewisse lokale Berühmtheit. Politiker*innen, Presse, Interessent ${ }^{*}$ innen und Mieterbeiräte ${ }^{14}$ kamen aus ganz Wien, um sich über das Pilotprojekt zu informieren. Es wurde ein Praxisleitfaden für die Rahmenbedingungen und den Aufbau von Gemeinschaftsgärten im Wiener Gemeindebau erstellt (Emprechtinger et al. 2010) und den Mitarbeiter*innen der zuständigen gemeindenahen Organisation Wohnpartner ${ }^{15}$ vorgestellt. Eine Verbreitung vergleichbarer Gärten fand trotz der positiven Beurteilung des Pilotprojekts im Wiener Gemeindebau bislang jedoch nicht statt. Wohnpartner hat sich im Rahmen des sozialarbeiterischen Auftrages für ein gänzlich anderes Konzept entschieden. Die Organisation fördert die Errichtung von einzelnen, öffentlich zugänglichen Hochbeeten. ${ }^{16}$ Der Aufwand ist dabei für alle Seiten geringer, doch können damit die Qualitäten eines Gemeinschaftsgartens bei Weitem nicht erzielt werden. Es gibt bislang allerdings nur eine vergleichende Untersuchung in Form einer studentischen Abschlussarbeit. Gemeinschaftsgärten werden von Wohnpartner nur dann unterstützt, wenn sich bereits ein Verein vor Ort gegründet hat. Die Organisation selbst ergreift nicht die Initiative. Das birgt die Gefahr, dass weiterhin eher jene Menschen Zugang zu einem Garten bekommen, die bereits ohnehin über ein größeres soziales und kulturelles Kapital verfügen.

\section{Zusammenfassung}

In diesem Artikel zeigte ich, wie Gemeinschaftsgärten im sozialen Wohnbau in Wien entwickelt werden können und welche Tätigkeiten im Sinne einer vita activa damit verbunden sind. Es wurde deutlich, warum im Wiener Gemeindebau zwar aufgrund seiner Bewohner*innen- und Verwaltungsstruktur Bottomup-Initiativen nicht bzw. kaum stattfinden oder geför-

14 Gewählte Interessensvertretung der Gemeindebaumieter*innen.

15 Wohnpartner ist eine Organisation der Wohnservice Wien Ges.m.b.H. („Wohnservice Wien“), die im Auftrag der Stadt Wien eine kostenlose Serviceeinrichtung für alle Fragen rund ums Wohnen im Gemeindebau ist https://wohnpartner-wien.at/ueber-uns/wo-sie-uns-antreffen [29.11.2019].

16 Laut Auskunft von Wiener Wohnen gibt es aktuell in den 1800 städtischen Wohnhausanlagen in Wien vier Nachbarschaftsgärten und 345 öffentlich zugängliche Hochbeete an 110 Standorten. Sie werden von der Organisation Wohnpartner beraten und begleitet (außer der Nachbarschaftsgarten Roda-Roda, der autonom verwaltet ist), die Hochbeete sind alle von Wohnpartner begleitet. 
dert werden, dass sie aber sehr wohl möglich sind, wenn die initiale Aktivität gut organisiert wird und die anfänglichen Hürden professionell überwunden werden. So gesehen stellen der Gemeindebau und insbesondere seine funktionalistisch geprägten Abstandsgrünflächen zwischen den Zeilenbauten der 1950er- bis 7oer-Jahre aufgrund des großen Flächenangebots und der inhomogenen Bewohner*innenstruktur ein interessantes Potenzial für das urbane Gärtnern dar. Damit kann durchaus an die frühere Konzeption des Roten Wien mit Selbstverwaltung und Gemeinschaftsaktivitäten angeknüpft werden. Eine geeignete Umsetzungsstrategie besteht im Wesentlichen aus zwei Prinzipien: (1) einer möglichst stabilen räumlichen, sozialen, ökonomischen und legistischen Rahmenstruktur, die an sich verändernde Gegebenheiten angepasst werden kann und (2) einem ernst gemeinten Partizipationsprozess, der auf Autonomie der neuen Gemeinschaft abzielt und schrittweise die lokalen Akteur*innen dazu ermächtigt.

Die Menschen, die den Initiator*innen folgend das Gärtnern verwirklichen, können in ihrem Alltag alle drei Aspekte einer vita activa ausüben, ein aktives Leben mit Arbeiten, Herstellen und Handeln. Im Gemeinschaftsgarten können sie Lebensmittel produzieren (Arbeiten), Dinge erschaffen (Herstellen) und zusammen Entscheidungen treffen (Handeln). Es ist das Handeln, das Tätigsein unter, zwischen und mit anderen Menschen, das für Hannah Arendt die höchste und laut ihrer Diagnose in der Konsumgesellschaft am wenigsten ausgeübte menschliche Tätigkeit ist. Handelnd und sprechend stellen sich die Menschen (hier vor allem Frauen) in ihrer Einzigartigkeit dar, haben Teil an der (Garten-) Welt und teilen miteinander, was das Leben so mit sich bringt. Dabei nimmt die Sprache eine wichtige Rolle ein und es wird verständlich, warum die Gärtner*innen einerseits darum ringen, trotz der mitunter fehlenden gemeinsamen Sprache Verständigung herzustellen und Handeln zu ermöglichen und warum andererseits nicht alle Gärtner*innen tatsächlich handelnd tätig werden (können). Doch generieren sie gemeinsam Macht, indem sie jährlich einen Vorstand wählen - allerdings überwiegend Frauen mit österreichischer Muttersprache und ohne Doppel- und Mehrfachbelastung -, dem sie die Verantwortung zuerkennen und der den Großteil der Verantwortung für die nach außen selbstbestimmte Gartengemeinschaft trägt.

Die Eigenverantwortung für den Freiraum steht im Kontrast zu den leitenden Prinzipien der paternalistisch orientierten Verwaltung der Wohnhausanlagen. Und doch ist es gelungen, die durch das funktionalis- tische Leitbild entstandenen flächenintensiven Strukturen $\mathrm{zu}$ nutzen und durch gemeinsames Gärtnern „umzubrechen“. Mit dem Gemeinschaftsgarten konnte im funktionellen Geschoßwohnungsbau ein zwar unvollständiges, aber dafür gemeinschaftliches Außenhaus geschaffen werden. Die noch fehlenden Elemente eines Außenhauses, jene direkt mit der Wohnung verbundenen Bereiche, sind den Bewohner*innen durch den Garten bewusster geworden. Seit Errichtung des Gemeinschaftsgartens sind in dieser Wohnhausanlage an vielen Stellen von Mieter ${ }^{\star}$ innen gepflanzte Beete vor Balkonen und Haustüren aufgetaucht. Sie werden bislang toleriert und regen die Verwaltung zu neuen Formen des Umgangs mit Eigeninitiative an. Die Konfliktlinie zwischen der Eigenmacht der Bewohner*innen und der wohlfahrtsstaatlichen, zentralistischen Verwaltung wird damit weiter aufgeweicht und neue Handlungsspielräume können entstehen.

\section{Literatur}

Appel, I./Grebe, C./Spitthöver, M. (2011): Aktuelle Garteninitiativen: Kleingärten und neue Gärten in deutschen Großstädten. Kassel: kassel university press.

Arendt, H. (1969): Vita activa oder Vom tätigen Leben. München, Zürich: Piper. 12. Auflage.

Arendt, H. (2013): The human condition. Chicago: University of Chicago Press.

Armstrong, D. (2000): A survey of community gardens in upstate New York: Implications for health promotion and community development. Health \& place, 6 (4), 319-327.

Arnstein, S.R. (1969): A ladder of citizen participation. Journal of the American Institute of planners, 35 (4), 216-224.

Auböck, M. (1975): Die Gärten der Wiener. Wien: Jugend u. Volk.

Bahrdt, H.P. (2013): Die moderne Großstadt: Soziologische Überlegungen zum Städtebau. Wiesbaden: Springer Verlag.

Bennholdt-Thomsen, V. (2014): A Subsistence Perspective for the Transition to a New Civilization: An Ecofeminist Contribution to Degrowth. Canadian Woman Studies, 31 $(1 / 2), 20-26$.

Blau, E. (1999): The Architecture of Red Vienna, 1919-1934. London: Mit Press.

Bobek, H./Lichtenberger, E. (1966): Wien: Bauliche Gestalt und Entwicklung seit der Mitte des 19. Jahrhunderts. Graz: Böhlau.

Böse, H. (1981): Das Außenhaus verfügbar machen. In: AG Freiraum und Vegetation (Hg.): Notizbuch 10 der Kasseler Schule. Kassel: Gesamthochschule Kassel, 52-6o.

Cumbers, A./Shaw, D./Crossan, J./McMaster, R. (2018): The Work of Community Gardens: Reclaiming Place for 
Community in the City. Work, Employment and Society, 32 (1), 133-149.

Czeike, F. (2004): Historisches Lexikon Wien: Ru-Z. Wien: Kremayr \& Scheriau.

Drummond, J.S./Themessl-Huber, M. (2007): The cyclical process of action research: the contribution of Gilles Deleuze. Action Research, 5 (4), 430-448.

Emprechtinger, J./Förster, K./Gruber, S./Mayrhofer, R./Staller, S./Stoik, C./Studer H. (2010): Wissenschaftliche Begleitforschung zur Einführung von Nachbarschaftsgärten im Wiener Gemeindebau. Wien: MA 50.

Exner, A./Kumnig, S./Krobath, P./Schützenberger, I./Brand, U. (2016): Stadtentwicklung, urbane Landwirtschaft und zivilgesellschaftlich gestalteter Grünraum in Wien. In: Fritz J./ Tomaschek, N. (Hg.): Gesellschaft im Wandel: Gesellschaftliche, wirtschaftliche und ökologische Perspektiven. Göttingen: Waxmann, 247-259.

Exner, A./Schützenberger, I. (2018): Creative Natures. Community gardening, social class and city development in Vienna. Geoforum, 92, 181-195.

Farkas, R. (1999): Zur Geschichte der Gartenbewegung im deutschsprachigen Raum. Bayerische Akademie für $\mathrm{Na}$ turschutz und Landschaftspflege, 23, 133-143.

Ferris, J./Norman, C./Sempik, J. (2001): People, land and sustainability: Community gardens and the social dimension of sustainable development. Social Policy \& Administration, 35 (5), 559-568.

Flick, U. (2011): Triangulation. In: Oelerich, G./Hans-Uwe Otto, H.-U. (Hg.): Empirische Forschung und Soziale Arbeit. Ein Studienbuch. Wiesbaden: Springer, 323-328.

Follmann, A./Viehoff, V. (2014): A green garden on red clay: creating a new urban common as a form of political gardening in Cologne, Germany. Local Environment, 20 (10), 1148-1174.

Förster, K. (2006): Vom Garten mit Häuschen zum Haus mit Gärtchen: Abschaffung der Kleingärten in Wien. Gärten als Handlungsfreiräume. Zur Organisation und Qualität von Freiräumen im Garten. Wien: Institut für Landschaftsplanung. Universität für Bodenkultur, 117-16o.

Fox-Kämper, R./Wesener, A./Münderlein, D./Sondermann, M./McWilliam, W./Kirk, N. (2017): Urban community gardens: An evaluation of governance approaches and related enablers and barriers at different development stages. Landscape and Urban Planning, 170, 59-68.

Frei, A.G. (1984): Rotes Wien: Austromarxismus und Arbeiterkultur: Sozialdemokratische Wohnungs-und Kommunalpolitik, 1919-1934. Wien: DVK Verlag.

Fürst, D. (2007): Urban Governance. Einblicke in die Diskussion. Städte im Umbruch - Das Online Magazin für Stadtentwicklung, Stadtschrumpfung, Stadtumbau \& Regenerierung, 4, 5-10.

Glover, T.D./Parry, D.C./Shinew, K.J. (2005): Building relationships, accessing resources: Mobilizing social capital in community garden contexts. Journal of Leisure Research, 37 (4), 450-474.
Hanisch, R./Widrich, M. (1999): Architektur der Küche. Zur Umwertung eines Wirtschaftsraums in der europäischen Architektur des zwanzigsten Jahrhunderts. In: Miklautz, E./Lachmayer, H./Eisendle, R. (Hg.): Die Küche. Zur Kulturgeschichte eines architektonischen, sozialen und imaginativen Raums. Wien, Köln, Weimar: Böhlau, 17-47.

Hilpert, T. (1978): Die funktionelle Stadt. Le Corbusiers Stadtvision-Bedingungen. Braunschweig: Vieweg.

Hilpert, T. (1984): Le Corbusiers „Charta von Athen“: Texte und Dokumente; kritische Neuausgabe. Braunschweig: Vieweg.

Hirsch, J. (1985): Fordismus und Postfordismus: Die gegenwärtige gesellschaftliche Krise und ihre Folgen. Politische Vierteljahresschrift, 26 (2), 160-182.

Homann, K./Jäger, A./Spitthöver, M. (2002): Freiraumqualität statt Abstandsgrün. 2. Perspektiven und Handlungsspielräume für Freiraumkonzepte mit hohem sozialem Gebrauchswert im Mietgeschosswohnungsbau. Kassel: Kassel University Press.

Hülbusch, I.-M. (1978): Innenhaus und Außenhaus - umbauter und sozialer Raum. Kassel: Gesamthochschule Kassel.

Jacobs, J. (1993): The death and life of great American cities. New York: Modern Library.

Kaplan, R./Kaplan, S. (1989): The experience of nature: A psychological perspective. CUP Archive.

Knecht, U./Krüger, C./Markert, D./Moser, M./Mulder, A.-C./ Praetorius, I./Roth, C./Schrupp, A./Trenkwalder-Egger, A. (2015): $A B C$ des guten Lebens. Rüsselsheim: Christel Göttert Verlag.

Kölzer, A. (2003): Wurzeln im Alltäglichen. Die Bedeutung der Arbeit am Symbolischen für eine Subsistenzperspektive in der Landschafts- und Freiraumplanung, dargestellt am Beispiel der Kasseler Erlenfeldsiedlung. Dissertation. Wien.

Krasny, E. (2012): Hands-on urbanism 1850-2012. Vom Recht auf Grün. Wien: Turia und Kant.

Kurowski, M. (2003): Freiräume im Garten: Die Organisation von Die Handlungsfreiräumen in der Landschafts- und Freiraumplanung. Dissertation. Wien.

Kurtz, H. (2001): Differentiating multiple meanings of garden and community. Urban Geography, 22 (7), 656-670.

Lamnek, S./Krell, C. (2010): Qualitative Sozialforschung. Weinheim: Beltz.

Lewis, C.A. (1972): Public housing gardens - landscapes for the soul. Landscape for living 277-282. Washington, D.C.: USDA Yearbook of agriculture 1972.

Macias, T. (2008): Working toward a just, equitable, and local food system: The social impact of Community-Based agriculture. Social science quarterly, 89 (5), 1086-1101.

Madlener, N. (2009): Grüne Lernorte. Gemeinschaftsgärten in Berlin. Würzburg: Ergon.

Marchart, P. (1984): Wohnbau in Wien, 1923-1983. Wien: Compress. 
Marquardt, B. (2002): Gemeineigentum und Einhegungen zur Geschichte der Allmende in Mitteleuropa. Berichte $\operatorname{der} A N L, 26,14-23$.

Mayrhofer, R. (2018): Co-Creating community gardens on untapped terrain - lessons from a transdisciplinary planning and participation process in the context of $\mathrm{mu}-$ nicipal housing in Vienna. Local Environment, 23 (12), 1207-1224.

McCormack, L.A./Laska, M.N./Larson, N.I./Story, M. (2010): Review of the nutritional implications of farmers' markets and community gardens: a call for evaluation and research efforts. Journal of the American Dietetic Association, 110 (3), 399-408.

McGlone, P. (1999): Food projects and how they work. York Publishing Services Limited for the Joseph Rowntree Foundation.

McNiff, J. (2013): Action research: Principles and practice. London: Routledge.

Miklautz, E./Lachmayer, H./Eisendle, R. (1999): Die Küche. Zur Geschichte eines architektonischen, sozialen und imaginativen Raums. Wien: Böhlau Verlag.

Müller, C. (2007): Intercultural gardens. Urban places for subsistence production and diversity. German Journal of Urban Studies, 46 (1), 1-6.

Müller, C. (2011): Urban Gardening: Über die Rückkehr der Gärten in die Stadt. München: Oekom.

Nettle, C. (2016): Community gardening as social action. London: Routledge.

Neurath, O. (1923): Österreichs Kleingärtner- und Siedlerorganisation. Wien: Kommissionsverlag der Wiener Volksbuchhandlung.

Novy, K./Förster, W. (1985): Einfach Bauen. Genossenschaftliche Selbsthilfe nach der Jahrhundertwende. Zur Rekonstruktion der Wiener Siedlerbewegung. Wien: Picus.

Ohmer, M.L./Meadowcroft, P./Freed, K./Lewis, E. (2009): Community Gardening and Community Development: Individual, Social and Community Benefits of a Community Conservation Program. Journal of Community Practice, 17 (4), 377-399.

Opll, F./Csendes, P. (2013): Wien-Geschichte einer Stadt: Band 3: Von 1790 bis zur Gegenwart. Wien: Böhlau Verlag.

Pudup, M.B. (2008): It takes a garden: Cultivating citizensubjects in organized garden projects. Geoforum, 39 (3), 1228-1240.

Reed, M.S. (2008): Stakeholder participation for environmental management: a literature review. Biological conservation, 141 (10), 2417-2431.

Reinprecht, C. (2007): 3. Social Housing in Austria. In: Scanlon, K./Whitehead, C. (Hg.): Social housing in Europe. London: London School of Economics and Political Science, 35-43.

Reinprecht, C./Datler G./Keckeis C./Kurtev A. (2011): Soziale Dynamiken im Stadtraum. Ein Projekt am Institut für Soziologie, Universität Wien im Auftrag der Stadt Wien (MA18).
Rosol, M. (2006): Gemeinschaftsgärten in Berlin: Eine qualitative Untersuchung zu Potenzialen und Risiken bürgerschaftlichen Engagements im Grünflächenbereich vor dem Hintergrund des Wandels von Staat und Planung. Berlin: Mensch \& Buch Verlag.

Saldivar-Tanaka, L./Krasny, M.E. (2004): Culturing community development, neighborhood open space, and civic agriculture: The case of Latino community gardens in New York City. Agriculture and Human Values, 21 (4), 399-412.

Schmelzkopf, K. (2002): Incommensurability, Land Use, and the Right to Space: Community Gardens in New York City1. Urban Geography, 23 (4), 323-343.

Shimpo, N./Stiles, R./Yokohari, M./Miyamoto, M. (2014): The Influence of the Viennese Allotment Gardens Act on the Housing Renovations in Kleingärten in Vienna, Austria. Urban and Regional Planning Review, 1 (o), 99-114.

Shinew, K.J./Glover, T.D./Parry, D.C. (2004): Leisure spaces as potential sites for interracial interaction: Community gardens in urban areas. Journal of Leisure Research, 36 (3), 336-355.

Sora (2008): Wohnqualität und soziale Gerechtigkeit in Wien: Ein Vergleich von Wohnqualität und Bewohnerstruktur zwischen 1995 und 2008. Online: https://www.wohnbauforschung.at/index.php?id=339 [24.09.2019].

Stadt-Wien (2018): Kleingärten - Statistiken. Online: https://www.wien.gv.at/statistik/lebensraum/kleingaerten/ [27.04.2018].

Statistik Austria (2009): EU-SILC 2008: Aktuelle Ergebnisse zum Auftakt des „Europäischen Jahres zur Bekämpfung von Armut und sozialer Ausgrenzung". Online: http://www.statistik.at/web_de/presse/o42559.html [24.09.2019].

Stout, M. (2010): Climbing the ladder of participation: Establishing local policies for participatory practice. Public Administration and management, 15 (1), 45-97.

Taborsky, U. (2008): Naturzugang als Teil des Guten Lebens: die Bedeutung interkultureller Gärten in der Gegenwart. Frankfurt am Main: Lang.

Till-Tentschert, U./Till, M./Eiffe, F./Glaser, T./Heuberger, R./ Kafka, E./Lamei, N./Skina-Tabue, M. (2011): Armutsgefährdungen und Lebensbedingungen in Österreich: Ergebnisse aus EU-SILC 2018. Sozialpolitische Studienreihe 5. Wien: ÖBV Verlag.

Turner, B. (2011): Embodied connections: sustainability, food systems and community gardens. Local Environment, 16 (6), 509-522.

Wagner, M. (1915): Das sanitäre Grün der Städte: Ein Beitrag zur Freiflächentheorie. Berlin: C. Heymann.

Wakefield, S./Yeudall, F./Taron, C./Reynolds, J./Skinner, A. (2007): Growing urban health: community gardening in South-East Toronto. Health promotion international, 22 (2), 92-101.

Whitehead, J. (1989): Creating a living educational theory from questions of the kind, ,How do I improve my practice?؛ Cambridge journal of Education, 19 (1), 41-52. 